\title{
Tolerance Analysis of Aligning an Astigmatic Laser Diode with a Single-Mode Optical Fiber
}

\author{
L. A. Wang and C. D. Su
}

\begin{abstract}
A general method of analyzing misalignment tolerance of coupling light from an astigmatic laser diode to a single-mode fiber is described. We apply the method to derive the $3 \mathrm{~dB}$ tolerances caused by longitudinal, lateral, and angular misalignments in the optimal conditions for two kinds of coupling schemes. One is by the use of a rotationally symmetric optical system, and the other by a rod which is a special case of utilizing a rotationally asymmetric optical system. In the latter coupling case, the misalignment caused by a laser is found to have a much less $3 \mathrm{~dB}$ tolerance in the vertical direction than that caused by a fiber.
\end{abstract}

Index Terms-Optical coupling, misalignment tolerance analysis.

\section{INTRODUCTION}

$\mathbf{H}$ IGH-coupling efficiency and large misalignment tolerances are two important optical requirements of packaging a laser diode for various applications in optical fiber communication. High-coupling efficiency implies an increase of the repeater spacing, and may lead to cost saving. For butt coupling, the coupling efficiency is very small, typically about $10 \%$, due to the mode mismatch between a laser diode and a single-mode fiber. The former generally has an elliptical beam profile owing to inherent astigmatism, while the latter has a circular profile. To enhance the coupling efficiency, the small laser's waist must be somehow transformed to match the large fiber's waist by, for example, utilizing a lens system. Many kinds of lens systems have been proposed to couple light from a laser diode to a single-mode fiber. These coupling methods can generally be divided into two groups. One group is to use discrete components as coupling optics, such as ball lenses [1], [2], GRIN lenses [3], cylindrical lenses [4], [5], and so on. The other is to integrate a lens with a single-mode fiber such as hemispherical lensed fibers [6]-[8], hyperbolic lensed fibers [9], [10], etc. Though coupling optics in these two groups may differ, the method of deriving a theoretical coupling efficiency by calculating the overlapping of two Gaussian beams is basically the same, and will be described in Section II.

Manuscript received March 22, 1996; revised August 26, 1996. This work was supported in part by the National Science Council, Taiwan, R.O.C., under Contract NSC83-0417-E002-005.

The authors are with the Institute of Electro-Optical Engineering, National Taiwan University, Taipei, Taiwan, R.O.C.

Publisher Item Identifier S 0733-8724(96)08912-8.
Large misalignment tolerances implies the positioning process in packaging a laser module can be greatly simplified, which may also lead to cost saving. W. B. Joyce et al. discussed the theoretical positioning tolerance of a singlemode fiber in coupling light from a circular laser beam [11]. In contrast, the positioning tolerance of an astigmatic laser diode has not been discussed, and will be dealt with in details in this paper. Two kinds of coupling schemes are considered. One is by employing a rotationally symmetric optical system, and the other by a cylindrical lens rod which may be considered as a special case of rotationally asymmetric optical systems. The optimal condition for light coupling utilizing a cylindrical lens was discussed in [4]. However, in Section III-B we point out the results derived there seem incorrect, and we therefore propose a new optimal condition.

The remaining paper is arranged as follows. In Section II, the derivation of a set of more general equations regarding the coupling efficiency of two Gaussian beams is reviewed. Two kinds of optics for laser-to-fiber coupling are discussed in Section III. First, a rotationally symmetric optical system is used for light coupling, and the emphasis is placed on the misalignment due to the astigmatic behavior of the laser diode. Second, a cylindrical rod lens is used for laser-tofiber coupling, and we derive the optimal coupling condition which is different from that reported in [4]. In addition, coupling efficiency and misalignment tolerances of the laser and the fiber are discussed in details. Finally, we conclude our investigation in Section IV.

\section{THEORETICAL BACKGROUND}

A general laser-to-fiber coupling system is shown in Fig. 1 where the field of a single-mode fiber is assumed symmetric, and can be described by a Gaussian beam with beam waist radius of $\omega_{o f}$. However, the field of a laser diode is assumed elliptical, and has Gaussian distributions in both vertical and horizontal directions with unequal beam waist radii of $\omega_{o x}$ and $\omega_{o y}$. The coupling efficiency of such system can be calculated by the overlapping integral of these two fields in any plane between the endfaces of the fiber and the laser. If one calculates the coupling efficiency in the plane 1 as depicted in Fig. 1. The more general coupling efficiency than that in [11] can be derived and expressed as

$$
\eta=\eta_{x} \eta_{y}
$$




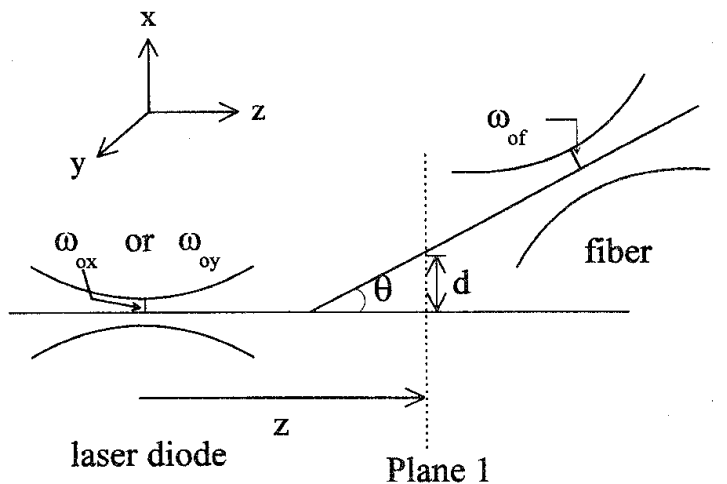

Fig. 1. Optical coupling between an astigmatic laser diode and a single-mode fiber.

where $\eta_{x}=\eta_{z}^{(x)} \eta_{d}^{(x)} \eta_{\theta}^{(x)} \eta_{d, \theta}^{(x)}$

$$
\begin{aligned}
& \eta_{z}^{(x)}= \\
& \frac{2}{\omega_{f} \omega_{x}} \frac{1}{\sqrt{\left(\frac{1}{\omega_{f}^{2}}+\frac{1}{\omega_{x}^{2}}\right)^{2}+\frac{k^{2}}{4}\left(\frac{1}{R_{x}}-\frac{1}{R_{f}}\right)^{2}}} \\
& \eta_{d}^{(x)}= \\
& \quad \exp \left\{-2 d^{2}\left[\frac{\frac{1}{\omega_{x}^{2} \omega_{f}^{2}}\left(\frac{1}{\omega_{x}^{2}}+\frac{1}{\omega_{f}^{2}}\right)+\frac{k^{2}}{4}\left(\frac{1}{\omega_{x}^{2} R_{f}^{2}}+\frac{1}{\omega_{f}^{2} R_{x}^{2}}\right)}{\left(\frac{1}{\omega_{x}^{2}}+\frac{1}{\omega_{f}^{2}}\right)^{2}+\frac{k^{2}}{4}\left(\frac{1}{R_{x}}-\frac{1}{R_{f}}\right)^{2}}\right]\right\}
\end{aligned}
$$$$
\eta_{\theta}^{(x)}=
$$$$
\exp \left\{-\frac{k^{2} \theta^{2}}{2} \frac{\frac{1}{\omega_{x}^{2}}+\frac{1}{\omega_{f}^{2}}}{\left(\frac{1}{\omega_{x}^{2}}+\frac{1}{\omega_{f}^{2}}\right)^{2}+\frac{k^{2}}{4}\left(\frac{1}{R_{x}}-\frac{1}{R_{f}}\right)^{2}}\right\}
$$$$
\eta_{d, \theta}^{(x)}=
$$$$
\exp \left\{k^{2} \theta d \frac{\frac{1}{R_{f} \omega_{x}^{2}}+\frac{1}{R_{x} \omega_{f}^{2}}}{\left(\frac{1}{\omega_{x}^{2}}+\frac{1}{\omega_{f}^{2}}\right)^{2}+\frac{k^{2}}{4}\left(\frac{1}{R_{x}}-\frac{1}{R_{f}}\right)^{2}}\right\} .
$$

Similarly, the expression of $\eta_{y}$ is obtained by replacing $x$ in (2)-(5) with $y$. In these equations, $\omega_{x, y}$ and $\omega_{f}$ are the beam's radii $R_{x, y}$ and $R_{f}$ are the radii of curvature of the laser and the fiber beams in plane 1 , respectively. All $\omega$ 's and $R$ 's are standard parameters of a Gaussian beam. $\omega$ is defined at where the intensity falls to $1 / e^{2} . k$ denotes the wave number, and $k=2 \pi / \lambda$ where $\lambda$ is the wavelength in free space. Coupling efficiencies $\eta_{x}$ and $\eta_{y}$ represent the contributions from $x$ and $y$ directions. $\eta_{z}^{(x)}$ denotes the coupling efficiency from $x$ direction without any kinds of misalignments. $\eta_{d}^{(x)}$ and $\eta_{\theta}^{(x)}$ indicate the coupling efficiencies with misalignments resulting from an offset $d$ and a tilt $\theta$ respectively, and $\eta_{d, \theta}^{(z)}$ indicates the combined effect of offset and tilt. In [11], the coupling efficiency component $\eta_{d, \theta}$ is presumably neglected, and the other components are basically equivalent to ours, although expressed in different forms. Note that the symmetry feature of coupling both Gaussian beams can be shown in these equations by simply interchanging between $R_{x, y}, \omega_{x, y}$ and $R_{f}, \omega_{f}$. In addition, to verify the above equations we also apply them to other coupling schemes described in literature. For instance, the coupling efficiency for a hemispherical lensed fiber can be obtained by substituting $R_{f}$ with $R_{f}=R_{L} /\left(n_{L}-1\right)$ where $R_{L}$ is the radius of the lens, and $n_{L}$ is its refractive index. The results are consistent with those obtained by Sakai et al. [7].

A special case is to find the coupling efficiency between two separate, symmetric Gaussian beams without any kinds of misalignments. Assume the beams have waist radii $\omega_{o 1}$ and $\omega_{o 2}$, and the distance between the two waists is $z$. Since the two beams are coaxial, the coupling efficiency can then be derived by employing (2). The resultant efficiency can be expressed as

$$
\eta=\frac{4 Z_{1} Z_{2}}{\left(Z_{1}+Z_{2}\right)^{2}+z^{2}}
$$

where $Z_{1}$ and $Z_{2}$ are Rayleigh ranges of two beams, and are defined as $Z_{1}=\pi \omega_{o 1}^{2} / \lambda$ and $Z_{2}=\pi \omega_{o 2}^{2} / \lambda$, respectively. Note that (6) is equivalent to (10) in [11]. Clearly, the maximum coupling efficiency occurs when the distance between the two beam's waists is zero, a case commonly denoted as butt coupling.

\section{OPTICAL COUPLING SCHEMES}

In this section, we will consider two optical coupling systems: rotationally symmetric, and rotationally asymmetric ones. In the rotationally symmetric system, we derive the tolerances when an astigmatic laser is misaligned with a single mode fiber. In the rotationally asymmetric system, we only consider a cylindrical lens as coupling optics because of its potential applications in coupling light from a laser array to a fiber array [13]. Coupling efficiencies and coupling losses due to various misalignments incurred either in the laser side or in the fiber side will be discussed separately.

\section{A. Rotationally Symmetric Optical System}

A system utilizing discrete optical components such as ball lens, GRIN lens and so on, or integrated components, for instance, hemispherical lensed fiber is by its nature of a rotationally symmetric optical system because these components possess rotational symmetry. In such system the coupling efficiency can be obtained by overlapping the laser and the fiber fields in the plane of the laser's endface. From Eq. (6), the maximum coupling efficiency occurs when the laser is placed in the image plane of the fiber

$$
\eta\left(\omega_{o f, i}\right)=\frac{2}{\frac{\omega_{o f, i}}{\omega_{o x}}+\frac{\omega_{o x}}{\omega_{o f, i}}} \times \frac{2}{\frac{\omega_{o f, i}}{\omega_{o y}}+\frac{\omega_{o y}}{\omega_{o f, i}}}
$$

where $\omega_{o f, i}$ is the beam waist radius of the fiber in the image plane. From (7), if $\omega_{o f, i}$ equals to the geometrical average of the laser's waist radii, i.e., $\omega_{o f, i}=\sqrt{\omega_{o x} \omega_{o y}}$, the optimal coupling efficiency is then achieved, and the result is consistent with that derived in [12].

When the laser and the fiber are placed in their optimal positions, the amounts of longitudinal $\left(z_{\ell}\right)$, lateral $\left(d_{\ell, x}, d_{\ell, y}\right)$ and angular $\left(\theta_{\ell, x z}, \theta_{\ell, y z}\right)$ misalignments of an astigmatic laser diode, which results in $3-\mathrm{dB}$ loss, can be calculated from (3), 
(4), and (6). To express the optimal coupling efficiency and the tolerances, we introduce an elliptical factor $\mathrm{m}$, which is defined as the ratio of the beam waist radii in the vertical and horizontal directions, i.e., $m=\omega_{o x} / \omega_{o y}$, then

$$
\begin{aligned}
\eta_{\text {opt }} & =\frac{4}{2+m+m^{-1}} \\
z_{\ell} & =Z_{\ell y}(m+1) \sqrt{\frac{\sqrt{m^{4}+14 m^{2}+1}-\left(m^{2}+1\right)}{2}} \\
d_{\ell, x} & =\omega_{o y} \sqrt{\frac{\ln 2}{2}\left(m+m^{2}\right)}, \\
d_{\ell, y} & =\omega_{o y} \sqrt{\frac{\ln 2}{2}(m+1)} \\
\theta_{\ell, x z} & =\frac{1}{k \omega_{o y}} \sqrt{2 \ln 2\left(\frac{1}{m}+\frac{1}{m^{2}}\right)} \\
\theta_{\ell, y z} & =\frac{1}{k \omega_{o y}} \sqrt{2 \ln 2\left(\frac{1}{m}+1\right)} .
\end{aligned}
$$

Figs. 2 and 3 show the coupling efficiency, longitudinal, lateral and angular misalignments of the laser versus the variation of $m$. Assume the lateral dimension of the active region is fixed, i.e., $\omega_{o y}$ is also fixed. When $m$ approaches one, i.e., the field becomes more circularly symmetric, the coupling efficiency is closer to the maximum value. The larger $m$ is, the smaller the divergence angle in the vertical direction becomes; while the larger the beam radius of the laser is in the vertical direction, the larger the corresponding Rayleigh range becomes. Hence, as $m$ increases, both longitudinal and lateral misalignment tolerances of the laser increase; the angular tolerance, however, decreases. The relatively small values of the lateral tolerance results from the small waist radii of the laser and the fiber (in the image plane). When there are some lateral misalignments, it is these small waist radii which leads to relatively less overlapping of the two fields. Similarly, it is noted that the lateral tolerance in the horizontal direction is greater than that in the vertical direction. Meanwhile, the divergence angle of the laser in the vertical direction is larger than that in the horizontal direction. Therefore, the angular misalignment tolerance of the laser in the vertical direction is greater than that in the horizontal direction.

\section{B. Coupling by a Cylindrical Lens-A Special Case of Rotationally Asymmetric Optical Systems}

A system utilizing optical components such as aspheric lenses does not possess rotational symmetry, and is termed as rotationally asymmetric. Here we only consider a special case of utilizing a cylindrical lens for light coupling, which may be regarded as one of the rotationally asymmetric optical systems.

A coupling configuration employing a cylindrical lens is schematically shown in Fig. 4 where $S_{o}$ is the distance between the laser diode and one vertex of the cylindrical lens, and $S_{i}$ is the distance between the fiber and the other vertex of the cylindrical lens. The cylindrical lens has power in the $x$ direction, but has no power in the $y$ direction. When it is used to couple light from a laser diode, the laser beam

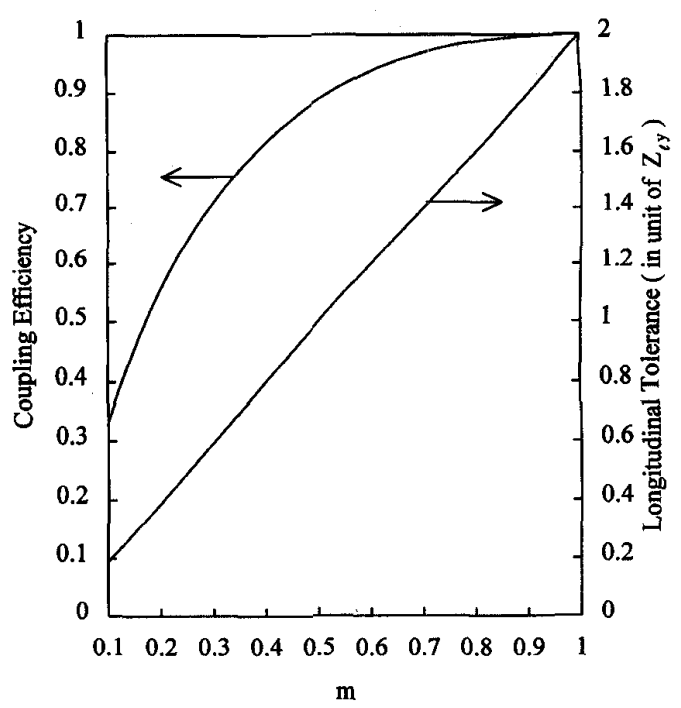

Fig. 2. Coupling efficiency and longitudinal tolerance versus elliptical factor $m$.

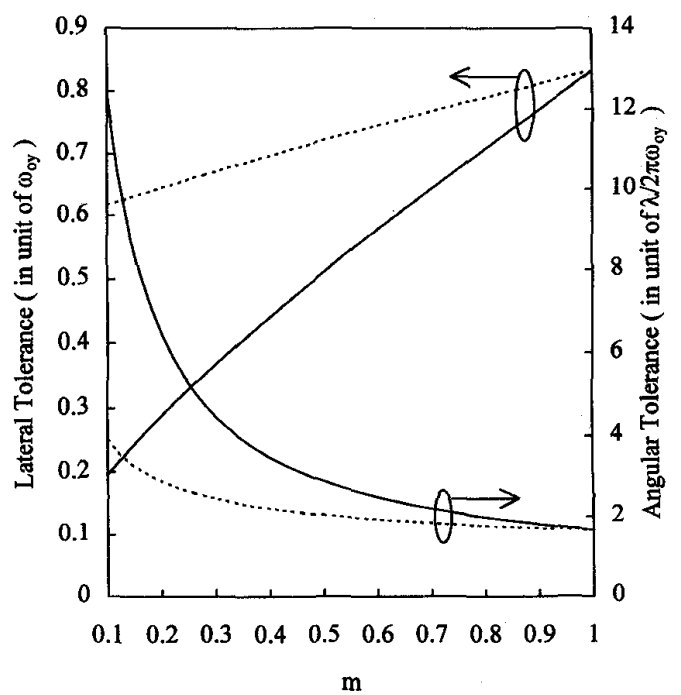

Fig. 3. Lateral and angular tolerances versus elliptical factor $m$. Solid curves for vertical direction and dash curves for horizontal direction.

after passing through the cylindrical lens will converge in one direction but will continue diverging in the other. Therefore, laser light with a symmetric beam profile propagating through a cylindrical lens will become asymmetric. Similarly, laser light with an asymmetric beam profile may become symmetric after a cylindrical lens. It is then possible to utilize a cylindrical lens to obtain a high coupling efficiency from an astigmatic laser diode.

In [4], Masatoshi et al. reported that the optimal coupling efficiency occurred when the fiber was attached to a cylindrical lens, that is $S_{i}=0$, and was also positioned at the image plane of the laser in the vertical direction. They then proceeded to find the distance between the laser and the cylindrical lens for optimizing the coupling efficiency. We find, however, the analysis is somewhat incorrect. The reason is the following: 


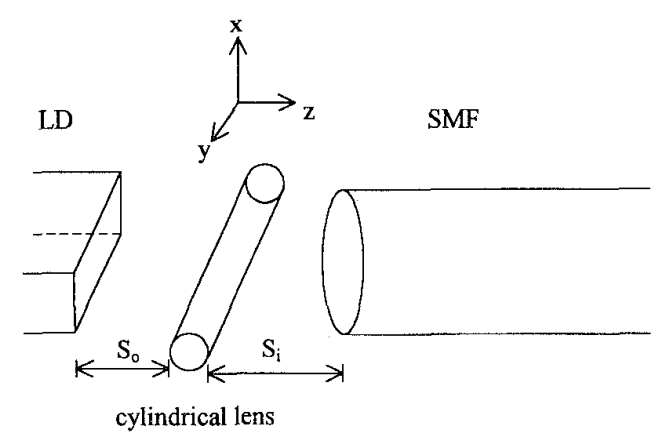

Fig. 4. Coupling configuration employing a cylindrical rod lens.

the conditions they found for the maximum coupling efficiency in the vertical direction should not be used to calculate the efficiency in the horizontal direction, and then to multiply the efficiencies in both directions together for the maximum total coupling efficiency. In fact, to find the optimal coupling efficiency, we should optimize the product of both efficiencies, not just one direction only.

We describe our new derivation of the optimal coupling efficiency as follows. To obtain the coupling efficiency, the overlapping integral of the laser and the fiber fields is calculated in the endface plane of the fiber. First, one can calculate the beam radii and the radii of curvature of the laser field in both horizontal and vertical directions obtained from the ABCD Law. Then, one can put these parameters in (1) to find the maximum overall coupling efficiency. It is found from such calculation that the maximum coupling efficiency occurs when the fiber is positioned as close contact with the cylindrical lens, that is $S_{i}=0$. It should be noted that in [4] the distance $S_{i}$ (or $z$ in their notation) was assumed, but not derived, to be null to proceed to find the maximum efficiency.

In the following, we will illustrate numerically an example of optical coupling by utilizing a cylindrical lens. An astigmatic laser diode having divergence angles of $10^{\circ}$ by $30^{\circ}$ at $1 / e^{2}$ intensity, and emitting wavelength at $1.3 \mu \mathrm{m}$ are assumed. The waist radius of the fiber's field is assumed $5 \mu \mathrm{m}$. Fig. 5 shows the coupling efficiency versus different radii of a cylindrical lens. From Fig. 5, the radius of a cylindrical lens needs to be small enough for high coupling efficiency. Such tiny cylindrical lenses with various radii can be fabricated, for example, by the method described in [13]. Therefore, in practice it is possible to use the cylindrical lens as the coupling optics to enhance light coupling from an astigmatic laser to a single-mode fiber. The relationship between the coupling efficiency and $S_{o}$ is depicted in Fig. 6 in which a cylindrical lens is assumed to have $20 \mu \mathrm{m}$ in diameter rather than the optimum value for the highest efficiency because of the consideration of relaxed fabrication. It is noted that there is an optimal distance $S_{o}$, about $5.0 \mu \mathrm{m}$ as shown in Fig. 6 . At the optimal distance, the laser beam's waist radii are close to the fiber's ones, thus resulting in a low coupling loss. Fig. 7 shows the relationship between the coupling efficiency and $S_{i}$. It is noted that the longitudinal tolerance of the fiber is quite large $(\sim 80 \mu \mathrm{m})$. The reason behind this is that the divergence angle of a laser beam is reduced to a small value in the vertical

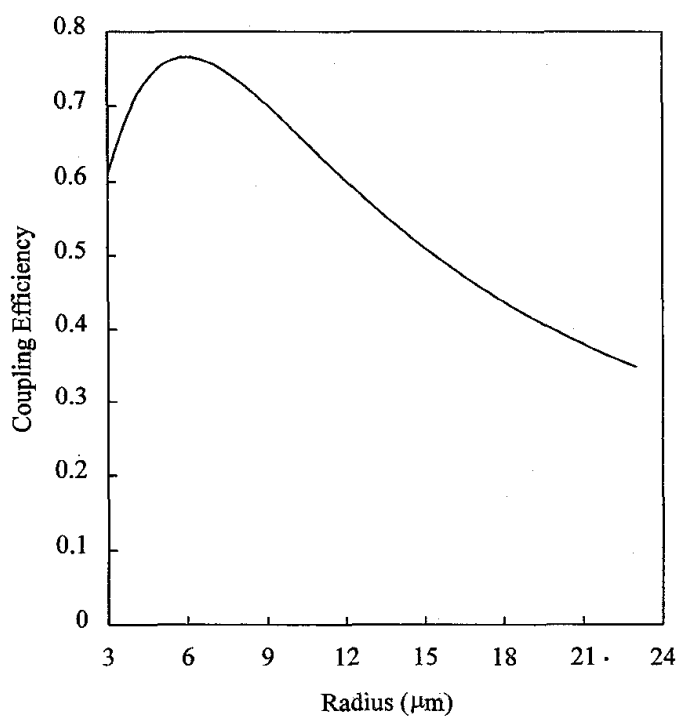

Fig. 5. Coupling efficiency versus radii of a cylindrical rod lens.

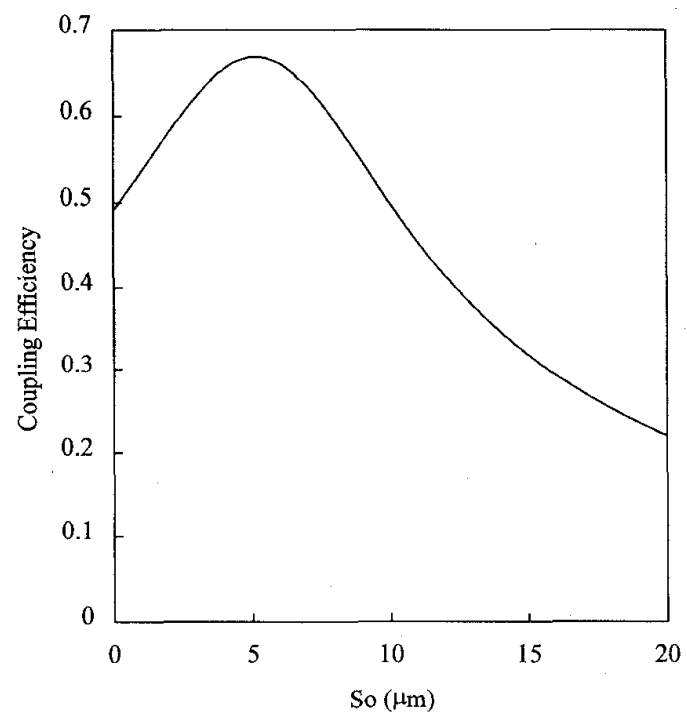

Fig. 6. Coupling efficiency versus $S_{o}$, rod radius $=10 \mu \mathrm{m}$.

direction after the beam propagates through a cylindrical lens. The smaller the divergence angle is reduced to, the larger the longitudinal tolerance becomes.

To calculate the losses due to the misalignments of a fiber, one can put the parameters of the laser field taken in the endface plane of the fiber into (3) and (4). Fig. 8 shows the normalized coupling efficiencies when both lateral and angular misalignments of the fiber are considered. The 3-dB lateral tolerances of the fiber are $\pm 5.6 \mu \mathrm{m}$ and $\pm 3.4 \mu \mathrm{m}$ in the vertical and in the horizontal directions, respectively. The $3-\mathrm{dB}$ angular tolerance of the fiber is $\pm 3.3^{\circ}$ in the vertical direction, and $\pm 5.8^{\circ}$ in the horizontal direction.

For comparison, we also calculate the losses due to the misalignments of a laser. In contrast to the previous situation, the overlapping of the laser and fiber fields is now performed in the endface plane of the laser. Similarly, one can calculate the 


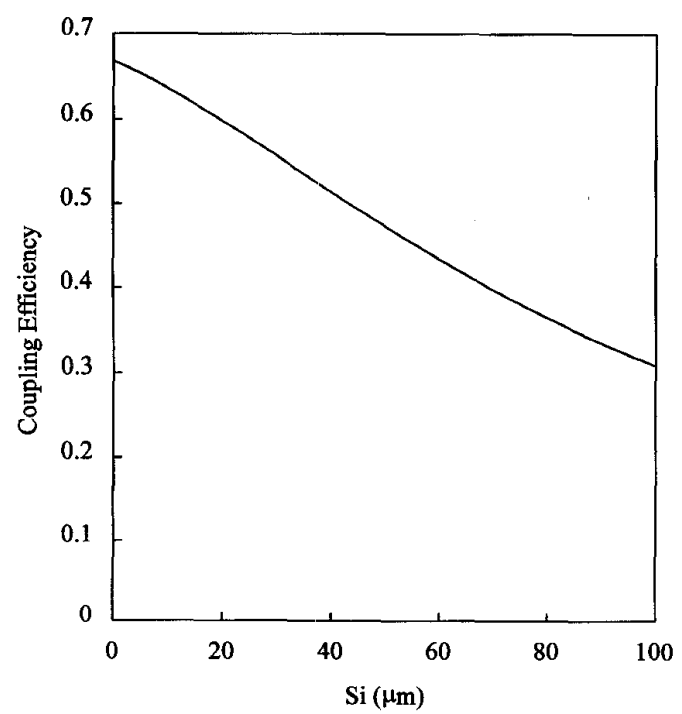

Fig. 7. Coupling efficiency versus $S_{i}$, rod radius $=10 \mu \mathrm{m}$.

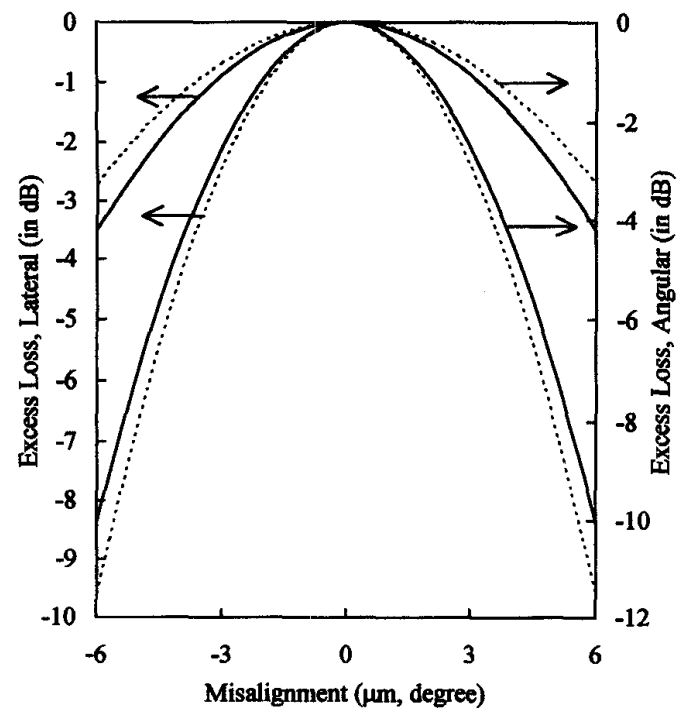

Fig. 8. Normalized coupling efficiencies versus lateral and angular misalignments of the fiber. Solid curves for vertical direction and dash curves for horizontal direction.

losses by first finding the field parameters of a fiber in the laser endface. Fig. 9 shows the normalized coupling efficiencies attributed to the lateral and the angular misalignments of the laser. The $3 \mathrm{~dB}$ lateral tolerances of the laser are $\pm 0.9 \mu \mathrm{m}$ and $\pm 3.4 \mu \mathrm{m}$ in the vertical and in the horizontal directions, respectively. The $3 \mathrm{~dB}$ angular tolerance of the laser is $\pm 20.7^{\circ}$ in the vertical direction, and $\pm 6.5^{\circ}$ in the horizontal direction.

It is noted that from Figs. 8 and 9, the $3 \mathrm{~dB}$ lateral tolerance is the same in the horizontal direction regardless of misalignment origins whether it is from the laser or from the fiber. This is because in the horizontal direction the relative position of the laser and the fiber is the same in both cases. However, the lateral tolerance in the vertical direction of the laser is much

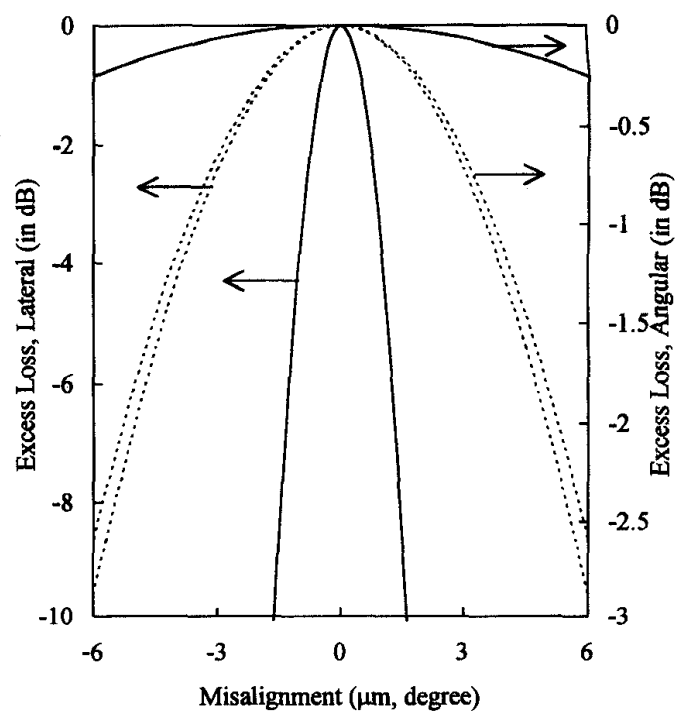

Fig. 9. Normalized coupling efficiencies versus lateral and angular misalignmentsof the laser. Solid curves for vertical direction and dash curves for horizontal direction.

smaller than that of the fiber because the beam radii of the two beams at the endface of the laser are smaller than those at the endface of the fiber. The angular tolerance of the laser is larger than that of the fiber since the divergence angle of the laser is larger than that of the fiber. If the misalignment cannot be eliminated then, from the above analysis, it is better to have the misalignments incurred by a fiber rather than by a laser. This is apparently because in the latter case, a severe, nonpractical tolerance requirement in the vertical direction should be met.

It is also observed that a cylindrical lens is more appropriately used for laser-to-fiber coupling when a laser has significantly different divergence angles in both horizontal and vertical directions. To demonstrate this feature, a cylindrical lens with $20 \mu \mathrm{m}$ diameter and a lasing wavelength of 1.3 $\mu \mathrm{m}$ are still assumed. Fig. 10 shows the relationship of the divergence angle and the coupling efficiency. In this case, we find that a cylindrical lens can offer relatively efficient optical coupling $(\eta>90 \%)$ when the divergence angles of a laser diode are in the range of $3^{\circ}-7^{\circ}$ and $12^{\circ}-28^{\circ}$ in the horizontal and in the vertical directions, respectively. For example, when the divergence angles of the laser are $5^{\circ}$ and $20^{\circ}$ in the vertical and horizontal directions, the coupling efficiency can reach as high as $98.2 \%$. In contrast, only $64 \%$ efficiency can be obtained when a rotational symmetric optical system is used for the same laser.

\section{CONCLUSION}

A general method to derive the efficiency of coupling light from an astigmatic laser diode to a single-mode fiber is reviewed. For a coupling optical system having rotationally symmetric properties, the $3 \mathrm{~dB}$ tolerances due to longitudinal, lateral, and angular misalignments of an astigmatic laser are expressed in simple forms under the optimal coupling conditions. As the laser beam becomes more circular, the coupling 


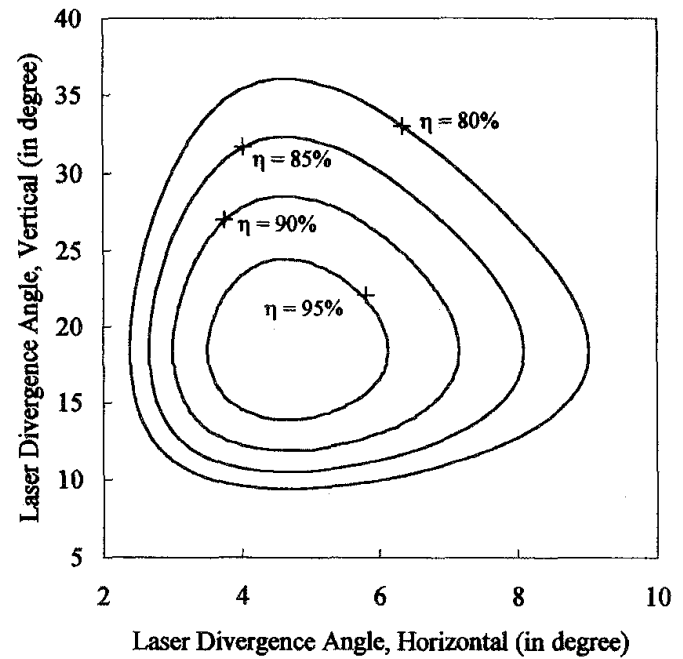

Fig. 10. Contour of coupling efficiency versus the divergence angles of a laser diode.

efficiency approaches $100 \%$, the longitudinal tolerance of the laser increases and approaches two times of the Rayleigh range, the lateral tolerance increases and approaches the beam radius of the laser, and the angular tolerance decreases. A cylindrical lens rod which is a special case of a rotationally asymmetric optical system is also analyzed, and the $3-\mathrm{dB}$ tolerances are derived. It is found that a cylindrical lens rod can have better coupling efficiency $(\eta>90 \%)$ than a rotationally symmetric lens when the range of divergence angle of a laser are $3^{\circ}-7^{\circ}$ in the vertical direction, and $12^{\circ}-28^{\circ}$ in the horizontal direction. In addition, it is found that the misalignment tolerances are relatively large except for the lateral tolerance of the laser in the vertical direction. Therefore, both high coupling efficiency and large tolerances can be achieved when using a cylindrical rod lens as coupling optics.

\section{REFERENCES}

[1] M. Sumida and K. Takemoto, "Lens coupling of laser diodes to singlemode fibers," J. Lightwave Technol., vol. LT-2, pp. 305-311, 1984.

[2] H. Karstensen and K. Drogemuller, "Loss analysis of laser diode to single-mode fiber couplers with glass spheres or silicon plano-convex lenses," J. Lightwave Technol., vol. 8, pp. 739-747, 1990.

[3] Y. Makita, I. Yamauchi, and K. Sono, "GRIN lenses for high efficiency coupling of laser diodes to single mode fiber," Fiber and Integrat. Opt., vol. 7, pp. $27-33,1988$.

[4] M. Saruwatari and K. Nawata, "Semiconductor laser to single-mode fiber coupler," Appl. Opt., vol. 18, pp. 1847-1856, 1979.

[5] E. Weidel, "New coupling method for GaAs-laser-fiber coupling," Electron. Lett., vol. 11, pp. 436-437, 1975.

[6] K. S. Lee and F. S. Barnes, "Microlenses on the end of single-mode optical fibers for laser applications," Appl. Opt., vol. 24, pp. 3134-3139, 1985.

[7] J.-I. Sakai and T. Kimura, "Design of a miniature lens for semiconductor laser to single-mode fiber coupling," IEEE J. Quantum Electron., vol. QE-16, pp. 1059-1067, 1980.

[8] J.-I. Yamada, Y. Murakami, J.-I. Sakai, and T. Kimura, "Characteristics of a hemispherical microlens for coupling between a semiconductor laser and single-mode fiber," IEEE J. Quantum Electron., vol. QE-16, pp. 1067-1072, 1980

[9] C. A. Edwards, H. M. Presby, and C. Dragone, "Ideal microlenses for laser to fiber coupling," J. Lightwave Technol., vol. 11, pp. 252-257, 1993.

[10] H. M. Presby and C. A. Edwards, "Near $100 \%$ efficient fiber microlenses," Electron. Lett., vol. 28, pp. 582-584, 1992.

[11] W. B. Joyce and B. C. DeLoach, "Alignment of Gaussian beams," Appl Opt., vol. 23, pp. 4187-4196, 1984.

[12] I. Ladany, "Laser to single-mode fiber coupling in the laboratory," Appl. Opt., vol. 32, pp. 3233-3236, 1993.

[13] L. A. Wang, J. Y. Hwang, M. S. Lin, and C. D. Su, "Electrical crosstalk and optical coupling for packaging a high-speed integrated DFB laser array," in Proc. Optic. Fiber Commun., paper WC1, 1995.

L. A. Wang, photograph and biography not available at the time of publication.

C. D. Su, photograph and biography not available at the time of publication. 